https://doi.org/10.15407/ujpe64.4.322

S. DZHUMANOV, I. KHIDIROV, U.T. KURBANOV, Z.S. KHUDAYBERDIEV, J.SH. RASHIDOV

Institute of Nuclear Physics, Uzbek Academy of Sciences

(Ulugbek, Tashkent 100214,Uzbekistan; e-mail: dzhumanov@inp.uz)

\title{
DISTINCTIVE FEATURES OF METAL-INSULATOR TRANSITIONS, MULTISCALE PHASE SEPARATION, AND RELATED EFFECTS IN HOLE-DOPED CUPRATES
}

\begin{abstract}
We study the distinctive features of the metal-insulator transitions, multiscale phase separation, and evolution of coexisting insulating and metallic/superconducting phases in hole-doped cuprates. We show how these interrelated phenomena and related effects manifest themselves in a wide doping range from the lightly doped to optimally doped regime in these systems, where the localized and mobile hole carriers reside in hole-poor (insulating) and hole-rich (metallic or superconducting) regions. We argue that small hole-rich regions (i.e. narrow nanoscale metallic islands or stripes) can persist in the insulating phase of the lightly doped cuprates, while the competing insulating, metallic, and superconducting phases would coexist in the underdoped cuprates. When the doping level is increased further, the hole-poor regions (or insulating zones) gradually narrow from macroscale to nanoscale insulating stripes and disappear in the optimally doped cuprates. We demonstrate clearly that the metal-insulator transitions and the coexisting insulating and metallic/superconducting phases are manifested in the suppression of superconductivity in underdoped cuprates and in the different temperature-dependent behaviors of the magnetic susceptibility and c-axis resistivity of lightly to optimally doped cuprates.

Ke ywords: hole-doped cuprates, metal-insulator transitions, multiscale phase separation, doping-dependent electronic properties.
\end{abstract}

\section{Introduction}

The mechanisms of metal-insulator transitions, microscopic phase separation, and subsequent evolution of insulating and metallic/superconducting phases in hole-doped copper oxides (cuprates) have not yet been compeletely clarified. In the undoped cuprates, the Mott-Hubbard model is believed to be appropriate to describe the existence of insulating and metallic phases [1-3]. However, the hole-doped cuprates are much more complex systems $[1,4-6]$ than the Mott-Hubbard insulators, and the nature of the relevant charge carriers and metal-insulator transitions has not been well established, because these materials in the intermediate doping regime exhibit the unusual insulating, metallic, and superconducting states $[1,2,7,8]$. In the overdoped regime, they become usual (normal) metals, while the new insulating, metallic, and superconducting phases of lightly to optimally doped cuprates exist between the

(C) S. DZHUMANOV, I. KHIDIROV, U.T. KURBANOV, Z.S. KHUDAYBERDIEV, J.SH. RASHIDOV, 2019 antiferromagnetic (AF) insulator and normal metal phases. The lightly doped, underdoped, and optimally doped cuprates share the most intriguing insulating, metallic, and superconducting properties, which cannot be understood in terms of the existing theories of insulators, metals, and superconductors based on the standard Wilson and Mott-Hubbard band models $[1,9]$ and the Bardeen-Cooper-Schrieffer (BCS) model of superconductivity [10]. In these systems, the electronic inhomogeneity, charge segregation, and ordering play a major role in the microscopic phase separation and the formation of alternating insulating and metallic/superconducting stripes (or phases) [11-15]. Recent micro X-ray diffraction studies have provided the best evidence of the multiscale phase separation from nanoscale to microscale stripes [16]. There are also indications for the coexistence of insulating and metallic/superconducting phases in high- $T_{\mathrm{c}}$ cuprates [17-19] and for the manifestations of these coexisting phases in the normal and superconducting properties of lightly doped, underdoped, and optimally doped cuprates [19-21]. Ap-

ISSN 2071-0194. Ukr. J. Phys. 2019. Vol. 64, No. 4 
parently, the evolution of the insulating and metallic/superconducting phases in these materials are manifested in the temperature dependences of their magnetic susceptibility $\chi[22]$ and resistivity $\rho$ [23], as well as in the suppression of superconductivity (i.e. in the so-called $1 / 8$ anomaly) in the underdoped regime $[24,25]$. Despite the considerable theoretical effort (see Refs. [8, 26-29]), our understanding of the unusual behaviors of the underdoped and optimally doped cuprates both in the normal state (above the superconducting transition temperature $T_{\mathrm{c}}$ ) and in the superconducting state is still far from the satisfactory one. The main problem is that the insulating and metallic/superconducting properties of these materials depend on the nature and types of charge carriers introduced by the doping, which have especially been the subject of controversy, being attributed either to some hypothetical quasiparticles (e.g., holons and quasifree electrons or holes) $[2,30]$ or self-trapped quasiparticles (large and small polarons) [31-33]. The idea of phase separation and segregation of charge carriers in the form of insulating and metallic clusters or stripes in doped cuprates has a long history (see, e.g., Refs. [34-36]). This important idea was proposed very early $[34,35]$ and then pursued in different models $[12,13,15,37-39]$. The distinguished feature of the cuprate compounds is that they are typical polar materials. Therefore, the electron-phonon interactions in doped cuprates are expected to be sufficiently strong and unconventional. Apparently, doping-induced effects, unconventional electron-phonon interactions, and polaronic effects play an important role in these polar materials $[5,8,31,32,40]$ and create inhomogeneities, which may give rise to the phase separation into insulating and metallic/superconducting regions in the lightly to optimally doped cuprates. The distinctive features of the metal-insulator transitions and the multiscale phase separation, which are manifested in the dopingdependent electronic properties of the doped cuprates have not yet been studied thoroughly. So far, the understanding of the origin and the evolution of the insulating and metallic/superconducting phases in these intricate materials represents a formidable challenge for both theory and experiment (see, e.g., question marks in the phase diagrams of hole-doped cuprates presented in Refs. [41-43]).

In this work, we study the distinctive features of the metal-insulator transitions, multiscale phase sep- aration, and subsequent evolution of coexisting insulating and metallic/superconducting phases in holedoped cuprates. We will clarify how these interrelated phenomena in $L a$ - and $Y$-based cuprates can occur in a wide doping interval from lightly to optimally doped regime. Then we will examine thoroughly the possible effects of metal-insulator transitions, multiscale phase separation, and evolution of coexisting insulating and metallic/superconducting phases on the high- $T_{\mathrm{c}}$ superconductivity in underdoped cuprates and on the magnetic susceptibility and the $c$-axis resistivity of lightly to optimally doped cuprates. In so doing, we will show that the distinctive features of metal-insulator transitions, microscopic phase separation, and related effects manifest themselves in the doping-dependent electronic properties of hole-doped cuprates, particularly in the suppression of superconductivity in underdoped cuprates and in the temperature-dependent behaviors of the magnetic susceptibility and the $c$-axis resistivity in lightly to optimally doped cuprates.

\section{Relevant Charge Carriers and Their Specific Ordering in Hole-Doped Cuprates}

The parent undoped cuprates with charge-transfer (CT) gaps $\Delta_{\mathrm{CT}} \simeq 1.5-2.0 \mathrm{eV}[1,2]$ are the MottHubbard insulators. Upon the $p$-type doping (hole doping) of cuprates, the free holes introduced into the oxygen valence band interact with lattice vibrations, and they become self-trapped quasiparticles (polarons) in a deformable lattice. A large ionicity of the cuprates $\eta=\varepsilon_{\infty} / \varepsilon_{0} \ll 1$ (where $\varepsilon_{\infty}$ and $\varepsilon_{0}$ are the high-frequency and static dielectric constants, respectively) enhances the polar electron-phonon interaction and the tendency to the polaron formation [31-33]. In these systems, the conditions for the formation of (bi)polarons, as shown in Refs. [44-46], are much easier than in other non-polar compounds. Actually, the self-trapping of holes by now has been discovered in different classes of substances (including alkali halides $[47,48]$ and cuprates $[5,49])$. Here, we note that the quasifree electrons or holes can exist only in ordinary metals, monoatomic semiconductors (e.g., Si and Ge), and heavily overdoped cuprates. A doping charge carrier interacting with surrounding ions of the lattice and altering the equilibrium positions of these ions creates an attractive potential well for the self-trapping of such a carrier. As a re- 
sult, the ground-state energy is always lowered by this polaronic effect. The binding energy $E_{\mathrm{p}}$ of a polaron is associated with the energy lowering resulted from carrier's adjusting to ionic vibrations. Charge carriers in doped cuprates can also form large bipolarons, in which two carriers are bound within a common selftrapping potential well [31]. The binding energy $E_{b \mathrm{~B}}$ of a large bipolaron is determined from the relation $E_{b \mathrm{~B}}=\left|E_{\mathrm{B}}-2 E_{\mathrm{p}}\right|$, where $E_{\mathrm{B}}$ is the ground-state energy of a large bipolaron. Theoretical $[31-33,50]$ and experimental $[5,51]$ studies showed that the charge carriers in doped cuprates are polarons with effective masses $m_{\mathrm{p}} \simeq(2-3) m_{\mathrm{e}}[5,52,53]$, where $m_{\mathrm{e}}$ is the free electron mass.

We argue that the ground states of doping charge carriers in polar cuprates are their self-trapped (polaronic) states lying in the $\mathrm{CT}$ gap, and the states of quasifree holes in the oxygen valence band become the excited states of hole polarons. Therefore, the new insulating gap $\varepsilon_{g}$ in doped cuprates is nearly ten times smaller than the CT gap $\Delta_{\mathrm{CT}}[1,3]$. It is well known $[31,48]$ that the large- and small-polaron states in three-dimensional solids are separated by a potential barrier. If the height of a potential barrier is sufficiently high [54], the existence of small polarons in the bulk is unlikely. A self-trapped carrier is usually called a large polaron, when its size is larger than the lattice constant. The lattice constants of the cuprates are about (4-6) $\AA$, while the values of the radius of polarons presented in Ref. [5] are about (8-10) A. Further, the relatively small binding energies of polarons $E_{\mathrm{p}} \simeq 0.06 \mathrm{eV}$ and $E_{\mathrm{p}} \simeq 0.12 \mathrm{eV}$ were observed in optimally doped and underdoped cuprates, respectively [51]. These and other experiments [49] suggest that the relevant charge carriers in doped cuprates are large polarons.

In the recent experimental observations [55-57], giant phonon anomalies in underdoped cuprates confirm also a large electron-phonon interaction leading to the complex ionic displacement pattern associated with the charge-density-wave (CDW) formation. Those phonon anomalies are a reminiscence of the anomalous phonon softening and broadening effects, which are caused by the polaron formation. Therefore, the formation of a CDW in doped cuprates is none other than the polaron formation in a deformable lattice. Actually, the CDW associated with the lattice distortion is similar to the polaronic picture. The size distribution of CDWs and their spatial arrangement have been determined at the nanoscale and the mesoscale, using the scanning micro X-ray diffraction [16]. The CDW puddles, like the vapor bubbles in boiling water, as seen by this $\mathrm{X}$ ray diffraction [16], are very similar to the deformation clouds of polarons. The lightly doped and underdoped cuprates are inhomogeneous systems (where the dopants and charge carriers are distributed inhomogeneously), and they are more inhomogeneous than optimally doped cuprates [58]. One can assume that the charge carriers (i.e., hole polarons) in these systems segregate into carrier-rich and carrier-poor regions as a result of their specific ordering. We argue that the specific ordering of these charge carriers in carrier-poor and carrier-rich domains, just like the ordering of atoms in solids, results in the formation of simple cubic, body-centered cubic, and face-centered cubic superlattices with coordination numbers $z=6$, 8 , and 12 , respectively, and the formation of different energy bands of polarons in the CT gap of the cuprates. In the carrier-poor regions, a narrow polaronic band is formed inside the $\mathrm{CT}$ gap. In this case, the system becomes an insulator, where polaronic carriers become localized, and their hopping conductivity occurs within the narrow polaronic band. In contrast, the charge transport in a sufficiently broadened polaronic band (i.e. in carrier-rich regions) becomes metal-like. Similarly, the charge transport in the broadened impurity band becomes also metal-like at higher doping levels.

\section{Distinctive Metal-Insulator Transitions and Multiscale Phase Separation in Hole-Doped Cuprates}

Many experiments (see Ref. $[54,58]$ ) testify to the segregation of charge carriers in inhomogeneous holedoped cuprates into carrier-rich and carrier-poor regions. We imagine this physically as follows. As the doping increases toward the optimally doped region, the specific charge ordering and segregation lead to the formation of dynamic (metallic) and static (insulating) stripes in carrier-rich and carrier-poor regions, respectively. It is natural to assume that the different superlattices of polarons are formed at their inhomogeneous spatial distribution. When the carrierpoor and carrier-rich regions coexist in underdoped cuprates, the important question arises: how and at which doping level $n=n_{c}$ does the width of the

ISSN 2071-0194. Ukr. J. Phys. 2019. Vol. 64, No. 4 
polaronic band reach a critical value, above which the polaron transport becomes metallic, and a doped cuprate material can undergo a phase transition from an insulator to a metallic state? The criterion for such metal-insulator transition can be written in the form [54]

$\frac{E_{\mathrm{p}}}{\varepsilon_{\mathrm{F}}} \gtrsim 0.5 \frac{a_{\mathrm{p}}}{R_{\mathrm{p}}}$

where $a_{\mathrm{p}}$ is the lattice constant of large polarons, $R_{\mathrm{p}}$ is the radius of such polaron, $\varepsilon_{\mathrm{F}}=\hbar^{2}\left(3 \pi^{2} n\right)^{2 / 3} / 2 m_{\mathrm{p}}$ is the Fermi energy of large polarons.

Criterion (1) for a certain level of doping $n=n_{c}$ (where $n$ is the density of polaronic carriers) can be rewritten as

$x=x_{c}=\frac{n_{c}}{n_{a}}=\frac{1}{3 \pi^{2} n_{a}}\left[\frac{4 m_{\mathrm{p}} E_{\mathrm{p}} R_{\mathrm{p}}}{\hbar^{2} a_{\mathrm{p}}}\right]^{3 / 2}$,

where $n_{a}=1 / V_{a}$ is the density of host lattice atoms, and $V_{a}$ is the volume per $\mathrm{CuO}_{2}$ formula unit in the cuprates.

For the simple cubic, body-centered cubic, and face-centered cubic superlattices of polarons with $z=6, z=8$, and $z=12$, the lattice constants of non-overlapping polarons can be determined as $a_{\mathrm{p}}=2 R_{\mathrm{p}}($ for $z=6), a_{\mathrm{p}}=(4 / \sqrt{3}) R_{\mathrm{p}}($ for $z=8)$, and $a_{\mathrm{p}}=2 \sqrt{2} R_{\mathrm{p}}$ (for $z=12$ ). The maximum and minimum values of $x_{c}$ determined from relation (2) correspond to the simple cubic and face-centered cubic superlattices of polarons. Therefore, applying criterion (2) for metal-insulator transitions to the cases of simple cubic and face-centered cubic superlattices of polarons, we can write

$x_{c 1}=\frac{1}{3 \pi^{2} \hbar^{3} n_{a}}\left[2 m_{\mathrm{p}} E_{\mathrm{p}}\right]^{3 / 2}$

and

$x_{c 2}=\frac{1}{3 \pi^{2} \hbar^{3} n_{a}}\left[\sqrt{2} m_{\mathrm{p}} E_{\mathrm{p}}\right]^{3 / 2}$.

Now, we estimate $x_{c 1}$ and $x_{c 2}$ for $\mathrm{La}_{1-x} \mathrm{Sr}_{x} \mathrm{CuO}_{4}$ (LSCO) and $\mathrm{YBa}_{2} \mathrm{Cu}_{3} \mathrm{O}_{7-\delta}(\mathrm{YBCO})$ by taking $m_{\mathrm{p}}=$ $=(2.1-2.7) m_{\mathrm{e}}$ in $\operatorname{LSCO}[5,52,53]$ and $m_{\mathrm{p}}=(2.0$ 4.0) $m_{\mathrm{e}}$ in YBCO $[5,59]$. In so doing, we use the theoretical values of $E_{\mathrm{p}} \simeq(0.09-0.106) \mathrm{eV}$ at $\varepsilon_{\infty}=3.5$ and $\eta=0.02-0.10$ [54]. The values of $V_{a}$ are determined approximately as follows. The lattice constants of the orthorhombic LSCO can be taken approximately to be $a=b \simeq 5.4 \AA$ and $c \simeq 13 \AA$
[60]. Then the volume of the primitive unit cell of LSCO is about $380 \AA^{3}$, while the volume per $\mathrm{CuO}_{2}$ formula unit $V_{a}$ in LSCO is equal to $190 \AA^{3}$. Further, the lattice constants of $\mathrm{YBa}_{2} \mathrm{Cu}_{3} \mathrm{O}_{7-\delta}$ are taken approximately to be $a=b \approx 4 \AA$ and $c \approx 12 \AA$ [60], so that the volume per $\mathrm{CuO}_{2}$ formula unit in YBCO can be taken approximately to be $V_{a} \simeq 100 \AA^{3}$. Then we find $n_{a} \simeq 0.53 \times 10^{22} \mathrm{~cm}^{-3}$ (for LSCO) and $n_{a} \simeq$ $\simeq 10^{22} \mathrm{~cm}^{-3}$ (for YBCO). Using the above theoretical and experimental values of the parameter $m_{\mathrm{p}}, E_{\mathrm{p}}$ and $n_{a}$, we obtain the following values of critical dopings for metal-insulator transitions: $x_{c 1} \simeq 0.07-0.131$ and $x_{c 2} \simeq 0.042-0.078$ in LSCO and $x_{c 1} \simeq 0.035-$ 0.125 and $x_{c 2} \simeq 0.021-0.074$ in $\mathrm{YBCO}$.

Thus, we see that, in LSCO, the metal-insulator transitions and multiscale (from nanoscale to macroscale) phase separation into carrier-rich metallic (at $x>x_{c 1}$ and $x>x_{c 2}$ ) regions and carrier-poor insulating (at $x<x_{c 2}$ and $x<x_{c 1}$ ) regions may occur in the doping interval from $x \simeq 0.042$ (lightly doped region) to $x \gtrsim 0.131$ (underdoped region including also the "magic doping $x=1 / 8$ "), while such metalinsulator transitions and phase separation in YBCO would occur in the doping interval from $x \simeq 0.021$ to $x \simeq 0.125$. These results are in reasonable agreement with the experimental data on the metal-to-insulator crossover and the stripe formation in lightly doped and underdoped cuprates $[18,24,25,61,62]$.

\section{Manifestations of Metal-Insulator Transitions, Multiscale Phase Separation, and Related Effects in Hole-Doped Cuprates}

Attempts to explain the unusual normal-state properties of underdoped cuprates (in particular, insulatormetal crossover taking place far in the superconducting region, the insulating behavior of the $c$-axis resistivity, and the temperature-dependent magnetic susceptibility in the metallic state) and their suppressed superconductivity and pseudogap behaviors have led to many controversial assumptions (see, e.g., Refs. [1, 4, 19, 24, 25, 28]). One point of view, which stands out in the context of the experimental evidence, is the idea of preformed pairs [6] considered to be the fluctuating Cooper pairs, which are believed to be present in HTSC well above $T_{\mathrm{c}}$ [63]. Unconventional interactions between quasiparticles in underdoped cuprates may lead to their new and unidentified electronic states. Importantly, an abrupt change 
of the carrier velocity at $50-80 \mathrm{meV}$ has been observed by the angle-resolved photoemission spectroscopy (ARPES) in high- $T_{\mathrm{c}}$ cuprates [64]. Such kink-like feature in the electronic quasiparticle dispersions around $50-80 \mathrm{meV}$ cannot be explained by any known process other than a specific electron-phonon coupling. In reality, the unconventional electron-phonon coupling and polaronic effects are major factors influencing the electronic properties (in particular, magnetic susceptibility and resistivity) of lightly to optimally doped cuprates.

As is well known, the magnetic susceptibility and electric conductivity in the normal state of underdoped cuprates are different from the ones explained by the usual band theory. Although some researchers have started to construct the theory of magnetic susceptibility and charge transport in the cuprates [26$28,65]$, the magnetic susceptibility and the charge transport in the $c$-direction have seldom been investigated. We now discuss the problem of the insulatorto-metal crossover by studying the doping and temperature dependences of the magnetic susceptibility and the $c$-axis resistivity of lightly doped, underdoped, and optimally doped cuprates. In so doing, we use the large (bi)polaron model, the impurity and polaron band models. We also use the unusual form of a BCS-like pairing theory of polarons and believe that such modified BCS-like theory is applicable to the underdoped and optimally doped cuprates. In the following, we study the possible manifestations of the metal-insulator transitions and competing effects of coexisting insulating and metallic/superconducting phases in the temperature dependences of the magnetic susceptibility $\chi(T)$ and the $c$-axis resistivity $\rho_{c}(T)$ of lightly to optimally doped cuprates and in the suppression of superconductivity (i.e. $T_{\mathrm{c}}$ ) in underdoped cuprates with regard for the real physical situation in these systems.

\subsection{Supression of superconductivity in underdoped high-T $T_{\mathrm{c}}$ cuprates}

The above results provide a natural explanation for the so-called $1 / 8$ anomaly (i.e. the suppression of superconductivity at $x=1 / 8$ ) in the underdoped cuprates. According to our results, the doping level $x=1 / 8$ is of vital importance for various underdoped cuprates, since the part of dynamic stripes becomes static at $x \leq 1 / 8$ and metallic/superconducting domains begin to decrease just at this doping level.
The critical temperature of the superconducting transition $T_{\mathrm{c}}$ depends on the density $n_{s}$ of superfluid carriers (i.e. bosonic Cooper pairs) and reaches the maximum at the optimal doping. If the interboson coupling constant $\gamma_{\mathrm{B}}$ is small, the maximum value of $T_{\mathrm{c}}$ in optimally doped cuprates can be determined as the superfluid condensation temperature of attracting bosons from the relation [3]

$T_{\mathrm{c}}^{\max } \simeq T_{\mathrm{BEC}}\left[1+C_{0} \gamma_{\mathrm{B}} \sqrt{k_{\mathrm{B}} T_{\mathrm{BEC}} / \varepsilon_{\mathrm{BA}}}\right]$,

where $T_{\mathrm{BEC}}=3.31 \hbar^{2} n_{s}^{2 / 3} / k_{\mathrm{B}} m_{B}$ is the Bose-Einstein condensation temperature of an ideal Bose gas of Cooper pairs, $m_{B}$ is the mass of bosonic (polaronic) Cooper pairs, $k_{\mathrm{B}}$ is the Boltzmann constant, $\varepsilon_{\mathrm{BA}}$ is the cutoff parameter for the attractive part of the interboson interaction potential, $C_{0}=$ $=\pi^{3 / 2} / 3.912 \approx 1.422$, and $\gamma_{B} \ll 1$.

We now estimate the value of $T_{\mathrm{c}}^{\max }$ for optimally doped LSCO and YBCO. By taking $m_{B}=2 m_{\mathrm{p}}=$ $=5 m_{\mathrm{e}}, \gamma_{B}=0.3, k_{\mathrm{B}} T_{\mathrm{BEC}} / \varepsilon_{\mathrm{BA}}=0.1$, and $n_{s}=$ $=1.5 \times 10^{19} \mathrm{~cm}^{3}$ for LSCO, we find $T_{\mathrm{BEC}} \simeq 35.3 \mathrm{~K}$ and $T_{c}^{\max }=1.135 \times T_{\mathrm{BEC}} \simeq 40 \mathrm{~K}$. Using the values of parameters $m_{B}=4.5 m_{\mathrm{e}}, \gamma_{B}=0.3, k_{\mathrm{B}} T_{\mathrm{BEC}} / \varepsilon_{\mathrm{BA}}=$ $=0.1$, and $n_{s}=6 \times 10^{19} \mathrm{~cm}^{3}$, we obtain $T_{\mathrm{BEC}} \simeq$ $\simeq 81.5 \mathrm{~K}$ and $T_{\mathrm{c}}^{\max } \simeq 92.5 \mathrm{~K}$ for optimally doped YBCO.

If we now assume that two different kinds of charge carriers are present in underdoped high$T_{\mathrm{c}}$ cuprates, i.e., mobile hole carriers in superconducting stripes and immobile hole carriers in adjacent insulating stripes, we may be able to explain the suppression of high- $T_{\mathrm{c}}$ superconductivity naturally, as will be shown here. Actually, in underdoped cuprates, the insulating and metallic/superconducting stripes coexist at $x \lesssim 0.13$. As a result, $T_{\mathrm{c}}$ becomes less than $T_{\mathrm{c}}^{\max }$. Therefore, the actual critical temperature of the superconducting transition for the two-component stripe-phase system can be defined as

$T_{\mathrm{c}}=\frac{V_{\mathrm{S}}}{V_{\mathrm{S}}+V_{\mathrm{I}}} T_{\mathrm{c}}^{\max }$

where $V_{\mathrm{S}}$ is the total volume of the superconducting regions of the underdoped cuprate material, $V_{\mathrm{I}}$ is the total volume of the insulating regions of this material. It is reasonable to assume that the ratio $V_{\mathrm{I}} / V_{\mathrm{S}}$ for underdoped system LSCO is about 0.2 at $x \lesssim 0.13$. 

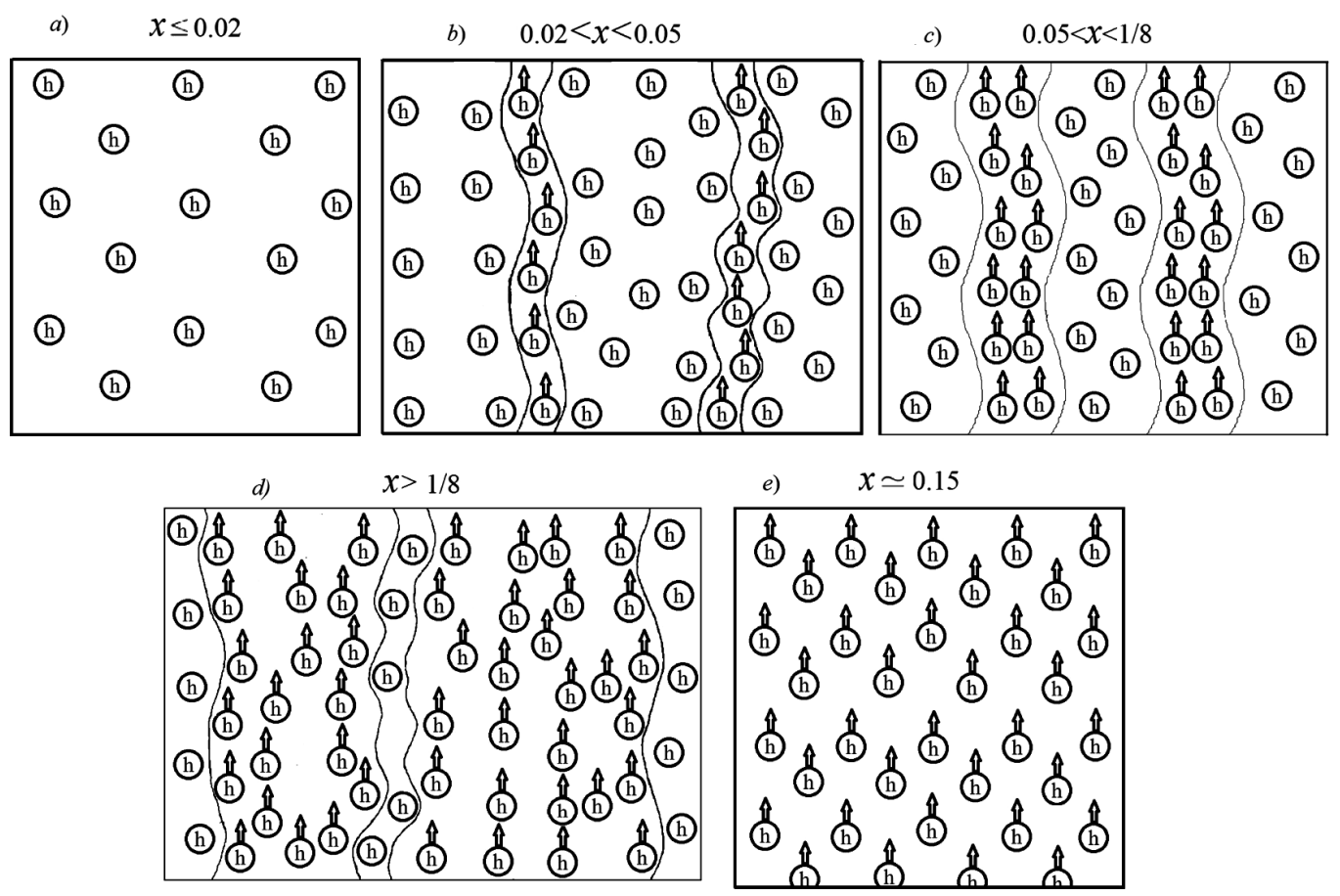

Fig. 1. Schematic picture of the metal-insulator transitions, multiscale phase separation from nanoscale to macroscale stripes, and evolution of coexisting insulating and metallic/superconducting phases (stripes) from lightly to optimally doped cuprates. Hole carriers (hole polarons) in the insulating phase of the lightly doped cuprates at $x \leq 0.02$ are immobile, as indicated without arrows $(a)$. Immobile and mobile hole carriers in the lightly doped cuprates $(0.02<x<0.05)$ are indicated, accordingly, in the wide insulating stripes without arrows and in the narrow metallic stripes with arrows $(b)$. Immobile and mobile hole carriers in the underdoped cuprates $(0.05<x<1 / 8)$ are indicated, accordingly, without and with arrows in insulating and metallic/superconducting stripes, which have comparable sizes $(c)$. Immobile and mobile hole carriers in the slightly underdoped cuprates $(x>1 / 8)$ are indicated, accordingly, in the narrow insulating stripes without arrows and in the wide metallic stripes with arrows $(d)$. Hole carriers in the metallic/superconducting phase of the optimally doped cuprates $(x \simeq 0.15)$ are indicated with arrows $(e)$

Then we find $T_{\mathrm{c}} \simeq 0.83 T_{\mathrm{c}}^{\max } \simeq 33 \mathrm{~K}$ for this system in accordance with the experiment (cf. the value of $T_{\mathrm{c}}=32 \mathrm{~K}$ at $x=0.13$ presented in Table 2 of Ref. [6]). For the underdped system YBCO, we can use the experimental value of $T_{\mathrm{c}}$ to determine the ratio $V_{\mathrm{I}} / V_{\mathrm{S}}$, by using Eq. (6). If we take $V_{\mathrm{I}} / V_{\mathrm{S}}=0.25$ for YBCO at $x=0.12$, we find $T_{\mathrm{c}}=T_{\mathrm{c}}^{\max } / 1.25=$ $=74 \mathrm{~K}$, which is consistent with the experimental data for YBCO [66] $\left(3 T_{\mathrm{c}} \simeq 220 \mathrm{~K}\right.$ at $\left.x=0.12\right)$. In $\mathrm{YBCO}$, the ratio $V_{\mathrm{I}} / V_{\mathrm{S}}$ is negligible at $x=1 / 8$. So, almost all of the hole carriers are superfluid carriers at $x \gtrsim 1 / 8$, and $T_{\mathrm{c}}$ changes little between $x \approx 0.125$ and $x_{\text {opt }} \approx 0.16$. These our predictions are also well consistent with the experimental results presented in Ref. [66].

Here, we propose (based on the above results) the microscopic picture of the phase separation from nanoscale to macroscale stripes and the evolution of coexisting insulating and metallic/superconducting phases in lightly to optimally doped cuprates (Fig. 1).

In this more realistic picture, the metallic phase is absent in the lightly doped cuprates $(x \simeq 0.02)$, in which the doped holes are immobile (Fig. 1, $a$ ) in a narrow polaronic band. Our results indicate that the first metallic phases appear in the lightly doped cuprates at $x>0.02$ in the form of narrow stripes (Fig. 1,b) in accordance with the experimental findings $[18,67]$. It follows that the insulator-to-metal transition and the nanoscale phase separation in the lightly doped cuprates $(x \simeq 0.02-0.05)$ occur only on a local scale (in small carrier-rich regions). Note that the metal-insulator transition occurring in these systems at $x=0.05$ and $T=0$ is manifested as a superconductor-insulator transition (see also 
Ref. [1]). For doping levels above $x=0.05$, the carrier-poor and carrier-rich regions as the insulating and metallic/superconducting phases coexist firstly in the form of competing static and dynamic stripes in the underdoped cuprates on a global (i.e. macroscopic) scale (Fig. 1, c). Then the insulating regions (or stripes) are gradually narrowed and finally disappear in the optimally doped cuprates. Thus, one can expect the coexistence of insulating and metallic/superconducting phases in lightly to optimally doped cuprates and the suppression of superconductivity (i.e., $T_{\mathrm{c}}$ ) in underdoped cuprates near the $x=1 / 8$ doping due to an increase of the insulating volume fraction at the expense of the superconducting volume fraction at $x<0.13$. Actually, in underdoped cuprates, the superconductivity is markedly suppressed, when the dynamic stripes become static at $x=1 / 8$ [25]. Finally, the insulating phases persist in the slightly underdoped cuprates in the form of narrow nanoscale stripes (Fig. 1, d), which would disappear in optimally doped cuprates (Fig. 1,e). The above multiscale phase separation from a local scale to a global scale occurs in lightly to optimally doped cuprates, as seen by the X-ray nanodiffraction in cuprates [16].

\subsection{Temperature dependence of the magnetic susceptibility in lightly doped cuprates}

In the lightly doped cuprates $(x \lesssim 0.05)$, both defect centers and polarons (which are products of the thermal dissociation of large bipolarons residing between the $\mathrm{CuO}_{2}$ layers) contribute to $\chi(T)$. In this case, the total dimensionless magnetic susceptibility can be determined from the relation

$$
\begin{aligned}
& \chi_{\mathrm{t}}(T)=\chi_{\mathrm{D}}(T)+\chi_{\mathrm{p}}^{*}(T)=\frac{\mu_{\mathrm{B}}^{2}}{k_{\mathrm{B}} T} n_{\mathrm{D}}- \\
& -2 \mu_{\mathrm{B}}^{2} \int_{0}^{\infty} D_{\mathrm{p}}(\varepsilon) \frac{\partial f_{\mathrm{p}}^{*}}{\partial \varepsilon} d \varepsilon,
\end{aligned}
$$

where $\chi_{\mathrm{D}}(T)$ and $\chi_{\mathrm{p}}^{*}(T)$ are the contributions to $\chi(T)$ coming from defect centers and polaronic carriers, respectively, $f_{\mathrm{p}}^{*}(\varepsilon)=\left[\exp \left(\left(\varepsilon+E_{b \mathrm{~B}}\right) / k_{\mathrm{B}} T\right)+1\right]^{-1}$ is the Fermi distribution function for the polarons produced by the thermal dissociation of bipolarons, $E_{b \mathrm{~B}}$ is the binding energy of a large bipolaron, $D_{\mathrm{p}}(\varepsilon)=$ $=\left(\sqrt{2} m_{\mathrm{p}}^{3 / 2} / \pi^{2} \hbar^{3}\right) \sqrt{\varepsilon}$ is the density of states of polarons, $\mu_{\mathrm{B}}$ is the Bohr magneton, and $n_{\mathrm{D}}$ is the density of defect centers.
Here, it is natural to believe that the density of defect centers $n_{\mathrm{D}}$ decreases with increasing the doping (i.e. in the metallic state), because the lightly doped cuprates are much more inhomogeneous in comparison with the optimally doped cuprates, as observed in experiments [58]. Further, we took into account that the magnitude of $E_{b \mathrm{~B}}$ varies from $0.0003 \mathrm{eV}$ to $0.014 \mathrm{eV}$ for $\varepsilon_{\infty}=3.5-4.5$ and $\eta=0.10-0.12$ [54]. The first term in Eq. (7) is nothing other than the Curie law $\chi_{\mathrm{D}}(T) \sim 1 / T$ for localized carriers, while the contribution of thermally dissociated bipolarons to $\chi(T)$ is

$\chi_{\mathrm{p}}^{*}(T)=\frac{2 \sqrt{2} \mu_{\mathrm{B}}^{2} m_{\mathrm{p}}^{3 / 2} \sqrt{k_{\mathrm{B}} T}}{\pi^{2} \hbar^{3}} \times$
$\times e^{E_{b \mathrm{~B}} / k_{\mathrm{B}} T} \int_{0}^{\infty} \frac{\sqrt{y} e^{y}}{\left(e^{\left(E_{b \mathrm{~B}} / k_{\mathrm{B}} T\right)} e^{y}+1\right)^{2}} d y$,

where $y=\varepsilon / k_{\mathrm{B}} T$.

Substituting Eq. (8) into Eq. (7), we define $\chi(T)$ for the cuprate material, which has the mass density $\rho_{\mathrm{M}}$, in the form

$$
\begin{aligned}
& \chi(T)=\frac{\chi_{t}(T)}{\rho_{\mathrm{M}}}=\frac{\mu_{\mathrm{B}}^{2}}{k_{\mathrm{B}} T \rho_{\mathrm{M}}}\left\{n_{\mathrm{D}}+\frac{2 \sqrt{2}\left(m_{\mathrm{p}} k_{\mathrm{B}} T\right)^{3 / 2}}{\pi^{2} \hbar^{3}} \times\right. \\
& \left.\times e^{E_{b \mathrm{~B}} / k_{\mathrm{B}} T} \int_{0}^{\infty} \frac{\sqrt{y} e^{y}}{\left[e^{\left(E_{b \mathrm{~B}} / k_{\mathrm{B}} T\right)} e^{y}+1\right]} d y\right\} .
\end{aligned}
$$

The variation of $\chi(T)$ in the lightly doped cuprate LSCO is shown in Fig. 2. The model fitting of experimental data for $\chi(T)$ is obtained, by using expression $(9)$, and is controlled mainly by the parameters $n_{\mathrm{D}}$ and $E_{b \mathrm{~B}}$. The best fit of the experimental $\chi(T)$ curve can be obtained under the following conditions: (i) the value of $n_{\mathrm{D}}$ in underdoped cuprates should be much smaller than in lightly doped cuprates; (ii) the value of $\varepsilon_{\mathrm{F}}$ should be larger than the value of $\varepsilon_{\mathrm{FI}}$, and (iii) the value of $m_{\mathrm{p}}$ should be much smaller than the value of $m_{\mathrm{I}}$. The value of the mass density $\rho_{\mathrm{M}}$ in the cuprates varies approximately from $4.0 \mathrm{~g} / \mathrm{cm}^{3}$ to $6.4 \mathrm{~g} / \mathrm{cm}^{3}$ [68]. The actual values of the effective polaron mass in these systems are about $m_{\mathrm{p}}=(2-5) m_{\mathrm{e}}$ $[5,69]$. As can be seen by the inspection of Fig. 2, $\chi(T)$ decreases with decreasing the temperature, reaches a minimum, and then increases exhibiting an insulating behavior in agreement with the experimental $\chi(T)$ curve for the lightly doped LSCO $(x=0.05)$ [22]. In Fig. 2, our theoretical curve is a much better fit of

ISSN 2071-0194. Ukr. J. Phys. 2019. Vol. 64, No. 4 
the experimental points, and expression (9) describes fairly well the insulating behavior of the magnetic susceptibility $\chi(T)$.

\subsection{Temperature dependence of the magnetic susceptibility in underdoped high- $T_{\mathrm{c}}$ cuprates}

When the densities of impurities (dopants) and polarons increase, the impurity and polaronic bands are formed in underdoped cuprates. It was argued [70] that the Cooper pairing without superconductivity may occur at low carrier concentrations in semiconductors. Similarly, the Cooper pairing of polarons at a characteristic temperature $T^{*}$ above $T_{\mathrm{c}}$ may occur in a degenerate polaronic Fermi gas with $\varepsilon_{\mathrm{F}} \gg k_{\mathrm{B}} T$, namely, in sufficiently broadened polaronic band [71], while the charge carriers in the narrow impurity band (i.e., charge carriers trapped by impurities) remain unpaired [72]. One can expect that the BCS-type Cooper pairing of large polarons occurs without superconductivity above $T_{\mathrm{c}}$ in carrier-rich regions of underdoped cuprates, while the real-space pairing of such polarons in carrier-poor regions leads to the formation of large bipolarons. Large bipolarons and polaronic Cooper pairs are dissociated into two separate polarons with increasing the temperature. Large bipolarons with binding energies $E_{b \mathrm{~B}} \ll 0.1 \mathrm{eV}$ can exist in carrier-poor (insulating) regions between the $\mathrm{CuO}_{2}$ layers, while the Cooper pairs of polarons can exist in carrier-rich (metallic) regions, where the Fermi energy of polarons is of order $\varepsilon_{\mathrm{F}} \gtrsim 0.1 \mathrm{eV}$. In underdoped cuprates, the defect centers, unpaired charge carriers in the impurity band, thermally dissociated large bipolarons, and polaronic Cooper pairs contribute to $\chi(T)$. Thus, the expression for the total dimensionless magnetic susceptibility in those materials can be written as

$\chi_{\mathrm{t}}(T)=\chi_{\mathrm{D}}(T)+\chi_{\mathrm{I}}(T)+\chi_{\mathrm{p}}^{*}(T)+\chi_{\mathrm{C}}(T)$,

where

$\chi_{\mathrm{I}}(T)=-2 \mu_{\mathrm{B}}^{2} \int_{0}^{\infty} D_{\mathrm{I}}(\varepsilon) \frac{\partial f_{\mathrm{I}}(\varepsilon)}{\partial \varepsilon} d \varepsilon$

is the contribution to $\chi(T)$ coming from the charge carriers in the impurity band, $D_{\mathrm{I}}(\varepsilon)=$ $=\left(\sqrt{2} m_{\mathrm{I}}^{3 / 2} / \pi^{2} \hbar^{3}\right) \sqrt{\varepsilon}$ is the density of states in the impurity band, $f_{\mathrm{I}}(\varepsilon)=\left[\exp \left(\left(\varepsilon-\varepsilon_{\mathrm{FI}}\right) / k_{\mathrm{B}} T\right)+1\right]^{-1}$ is the Fermi distribution function for the carriers in

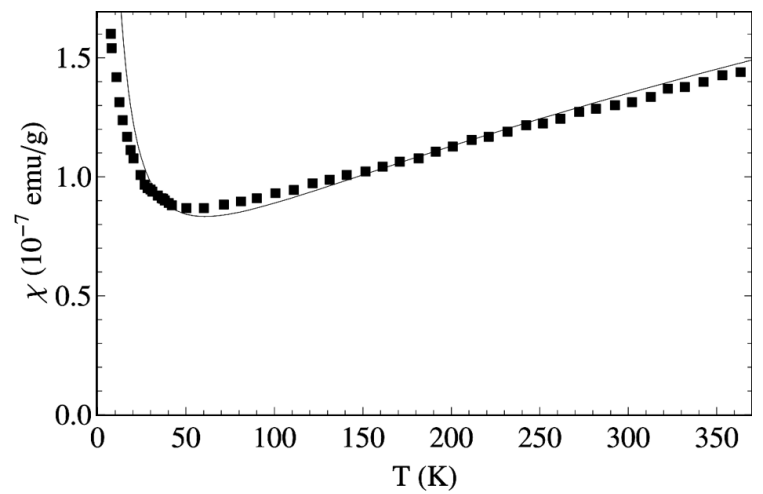

Fig. 2. Temperature dependence of $\chi$ in the lightly doped cuprate $\mathrm{La}_{2-x} \mathrm{Sr}_{x} \mathrm{CuO}_{4}(x=0.05)$ with the parameters $m_{\mathrm{p}}=$ $=4.3 m_{\mathrm{e}}, n_{\mathrm{D}}=0.145 \times 10^{20} \mathrm{~cm}^{-3}, \rho_{\mathrm{M}}=4.2 \mathrm{~g} / \mathrm{cm}^{3}$, and $E_{b \mathrm{~B}}=0.0018 \mathrm{eV}$. The theoretical results (solid curve) are compared with experimental data (ם) [22]

the impurity band (where the energy $\varepsilon$ of carriers is measured from the Fermi energy $\left.\varepsilon_{\mathrm{FI}}\right), m_{\mathrm{I}}$ is the mass of carriers in this band,

$\chi_{\mathrm{C}}(T)=-2 \mu_{\mathrm{B}}^{2} \int_{0}^{\infty} D_{\mathrm{c}}(\varepsilon) \frac{\partial f_{\mathrm{c}}(\varepsilon)}{\partial \varepsilon} d \varepsilon$

is the contribution to $\chi(T)$ coming from the polaronic components of the thermally dissociated Cooper pairs, $D_{\mathrm{c}}(\varepsilon)=D_{\mathrm{p}}(\varepsilon) / 2$ is the density of states at the Fermi surface for polarons of one spin orientation introduced in the BCS pairing theory [3], $f_{\mathrm{c}}(\varepsilon)=$ $=\left[\exp \left(\sqrt{\varepsilon^{2}+\Delta^{* 2}(T)} / k_{\mathrm{B}} T\right)+1\right]^{-1}$ is the Fermi distribution function for the excited polaron components of Cooper pairs, and $\Delta^{*}(T)$ is a BCS-like energy gap (or pseudogap) in the excitation spectrum of polaronic Cooper pairs.

Although the usual BCS theory of superconductivity in simple metals is inadequate for the explanation of high- $T_{\mathrm{c}}$ superconductivity in the underdoped cuprates, the unusual forms of Fermi-liquid theory and BCS-like pairing theory can be used to study the anomalous normal-state properties of high$T_{\mathrm{c}}$ cuprates. In particular, the modified BCS-like theory can adequately describe the Cooper pairing of polaronic carriers in the normal state of high- $T_{\mathrm{c}}$ cuprates above $T_{\mathrm{c}}$. The temperature dependence of the BCS-like gap parameter can be approximated as (cf. Refs. [73, 74])

$\Delta^{*}(T) \simeq 1.76 k_{\mathrm{B}} T^{*} \tanh \left[1.85 \sqrt{\frac{T^{*}}{T}-1}\right]$. 


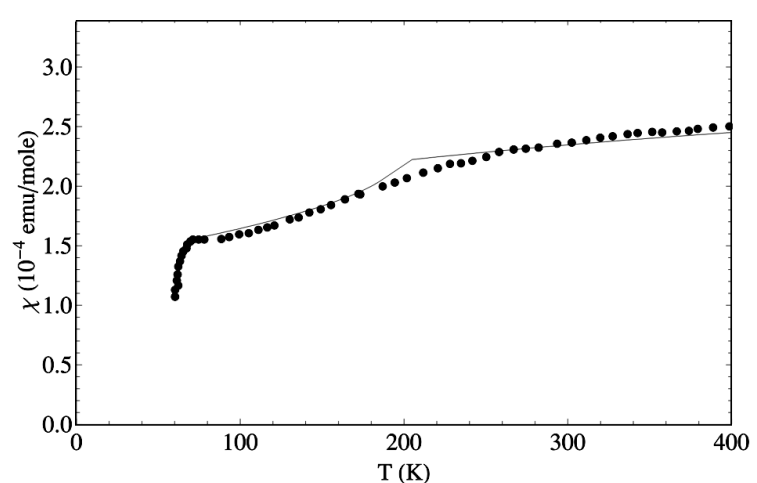

Fig. 3. Comparison of the theoretical results for the magnetic susceptibility of the high- $T_{\mathrm{c}}$ cuprate $\mathrm{YBa}_{2} \mathrm{Cu}_{3} \mathrm{O}_{6+x}$ (solid curve is obtained, by using the parameters $m_{\mathrm{p}}=3.0 m_{\mathrm{e}}$, $m_{\mathrm{I}}=4.7 m_{\mathrm{e}}, n_{\mathrm{D}}=0.08 \times 10^{19} \mathrm{~cm}^{-3}, \varepsilon_{\mathrm{F}}=0.145 \mathrm{eV}, \varepsilon_{\mathrm{FI}}=0.1$ $\mathrm{eV}, \rho=5.6 \mathrm{~g} / \mathrm{cm}^{3}$, and $E_{b \mathrm{~B}}=0.007 \mathrm{eV}$ ) with experimental data for $x=0.62(\bullet)[75]$

Here, we have compared numerically the BCS-like equation for $\Delta^{*}(T)$ and the simpler (i.e. convenient) expression (13) chosen by us for the calculation of $\Delta^{*}(T)$. In so doing, we checked that the analytical expression given by Eq. (13) is the best approximation to the BCS-like gap equation. One can assume that the main contribution both to $\chi_{\mathrm{C}}(T)$ and to BCS-like pairing of polarons below $T^{*}$ comes from polaronic carriers, whose energy is close to the Fermi energy $\varepsilon_{\mathrm{F}}$. Then $D_{\mathrm{c}}(\varepsilon)$ in Eq.(12) can be replaced by $D_{\mathrm{c}}\left(\varepsilon_{\mathrm{F}}\right)$. As a result, we obtain

$\chi_{\mathrm{C}}(T) \simeq 2 \mu_{\mathrm{B}}^{2} D_{\mathrm{c}}\left(\varepsilon_{\mathrm{F}}\right)\left[1+\exp \left(\Delta^{*}(T) / k_{\mathrm{B}} T\right)\right]^{-1}$.

Here, we note that $\varepsilon_{\mathrm{FI}}$ and $\varepsilon_{\mathrm{F}}$ are temperaturedependent, i.e.

$\varepsilon_{\mathrm{FI}}(T)=\varepsilon_{\mathrm{FI}}(0)\left[1-\left(\frac{\pi k_{\mathrm{B}} T}{12 \varepsilon_{\mathrm{FI}}(0)}\right)^{2}\right]$

and

$\varepsilon_{\mathrm{F}}(T)=\varepsilon_{\mathrm{F}}(0)\left[1-\left(\frac{\pi k_{B} T}{12 \varepsilon_{F}(0)}\right)^{2}\right]$.

In order to compare the calculated results for $\chi(T)$ with the experimental data [75], we write the final expression for $\chi(T)$ as

$\chi(T)=\chi_{\mathrm{t}}(T) \frac{M}{\rho_{\mathrm{M}}} \simeq \mu_{\mathrm{B}}^{2}\left\{\frac{n_{\mathrm{D}}}{k_{\mathrm{B}} T}+\right.$

$$
\begin{aligned}
& +\frac{2 \sqrt{2} m_{\mathrm{I}}^{3 / 2}}{\pi^{2} \hbar^{3} k_{\mathrm{B}} T} \int_{\varepsilon_{\mathrm{FI}}}^{\infty} \sqrt{\varepsilon} \frac{e^{\left(\varepsilon-\varepsilon_{\mathrm{FI}}(T)\right) / k_{\mathrm{B}} T}}{\left[e^{\left(\varepsilon-\varepsilon_{\mathrm{FI}}\right) / k_{\mathrm{B}} T}+1\right]^{2}} d \varepsilon+ \\
& +\frac{2 \sqrt{2} m_{\mathrm{p}}^{3 / 2}}{\pi^{2} \hbar^{3} k_{\mathrm{B}} T} \int_{0}^{\infty} \sqrt{\varepsilon} \frac{e^{\left(\varepsilon+E_{b \mathrm{~B}}\right) / k_{\mathrm{B}} T}}{\left[e^{\left(\varepsilon+E_{\mathrm{B}}\right) / k_{\mathrm{B}} T}+1\right]^{2}} d \varepsilon+ \\
& \left.+\frac{2 m_{\mathrm{p}}^{3 / 2}}{\sqrt{2} \pi^{2} \hbar^{3}} \frac{1}{\left(1+e^{\left.\Delta^{*}(T) / k_{\mathrm{B}} T\right)}\right.} \sqrt{\varepsilon_{\mathrm{F}}(T)}\right\} \frac{M}{\rho_{M}},
\end{aligned}
$$

where $M$ is the molar mass.

More importantly, the contribution of the last term in Eq. (17) becomes decisive for the fitting of experimental data below $T^{*} \simeq 200 \mathrm{~K}$. In the normal state of underdoped and optimally doped cuprates, the BCS-like pseudogap $\Delta^{*}(T)$ is manifested in $\chi(T)$ below $T^{*}$, as shown in Fig. 3 for the underdoped $\mathrm{YBa}_{2} \mathrm{Cu}_{3} \mathrm{O}_{6+x}$. At high temperatures $T \gg T^{*}$, the magnetic susceptibility of these high- $T_{\mathrm{c}}$ cuprates depends weakly on the temperature. According to (17), $\chi(T)$ begins to decrease gradually with lowering the temperature down to $T^{*}$. Then the decrease in $\chi(T)$ with lowering $T$ below $T^{*}$ becomes more rapid (see Fig. 3) due to the appearance of the pseudogap $\Delta^{*}$ in the excitation spectrum of the underdoped $\mathrm{YBa}_{2} \mathrm{Cu}_{3} \mathrm{O}_{6+x}$, for which we took the molar mass $M=668 \mathrm{~g} /$ mole [76] in our calculations.

The step-like kink in Fig. 3 is associated with the appearance of the BCS-like pseudogap near a characteristic temperature $T^{*}$ at the Cooper pairing of polarons. The expected step-like kink in the experimental $\chi(T)$ curve may be smeared out by simple inhomogeneities in the underdoped cuprates and was actually observed as a change or break of the slope of $\chi(T)$, by curving downward below $T^{*} \simeq 200 \mathrm{~K}$. In the experiment, the expected BCS-type (i.e. step-like) kink near $T^{*} \simeq 200 \mathrm{~K}$ is buried within the noises just like the BCS-like jump of the specific heat (see Ref. [72]) and observed as a mild pseudogap feature due to the sample inhomogeneity effects. When the inhomogeneity effects predominate over the pseudogap effect on $\chi(T)$, the BCS-like pseudogap feature near $T^{*}$ is strongly depressed or becomes less visible. In ordinary metals, $\chi(T)$ is temperature-independent due to the absence of a polaronic effect. In contrast, a polaronic signature appears in $\chi(T)$ both above $T^{*}$ and below $T^{*}$ in underdoped cuprates, where $\chi(T)$ firstly begins to decrease slowly with lowering the temperature down to $T^{*}$. Then the decrease in $\chi(T)$ with lowering $T$ below $T^{*}$ becomes more rapid (see Fig. 3 ). 


\subsection{Temperature dependence of the magnetic susceptibility in optimally doped high-T $T_{c}$ cuprates}

We now consider optimally doped YBCO, where the binding energy $E_{b \mathrm{~B}}$ of bipolarons residing between the $\mathrm{CuO}_{2}$ layers becomes vanishingly small, and the temperature $T^{*}$ is very close to $T_{\mathrm{c}}$. Here, the defect centers, charge carriers in the broadened impurity band (where $\varepsilon_{\mathrm{FI}}(T) \gg k_{\mathrm{B}} T$ and $D_{\mathrm{I}}(\varepsilon)$ is replaced by $\left.D_{\mathrm{I}}\left(\varepsilon_{\mathrm{FI}}(T)\right)\right)$, thermally dissociated large bipolarons, and polaron components of dissociated Cooper pairs contribute to the magnetic susceptibility of these high- $T_{\mathrm{c}}$ materials. The total dimensionless magnetic susceptibility in optimally doped cuprates can be written as

$$
\begin{aligned}
& \chi_{\mathrm{t}}(T)=\chi_{\mathrm{D}}(T)+\chi_{\mathrm{I}}(T)+\chi_{\mathrm{p}}^{*}(T)+\chi_{\mathrm{C}}(T)= \\
& =\mu_{\mathrm{B}}^{2}\left\{\frac{n_{\mathrm{D}}}{k_{\mathrm{B}} T}+\frac{2 \sqrt{2} m_{\mathrm{I}}^{3 / 2}}{\pi^{2} \hbar^{3} k_{\mathrm{B}} T} \sqrt{\varepsilon_{\mathrm{FI}}(T)}+\frac{2 \sqrt{2} m_{\mathrm{p}}^{3 / 2}}{\pi^{2} \hbar^{3} k_{\mathrm{B}} T} \times\right. \\
& \times \int_{0}^{\infty} \sqrt{\varepsilon} \frac{e^{\left(\varepsilon+E_{b \mathrm{~B}}\right) / k_{\mathrm{B}} T}}{\left[e^{\left(\varepsilon+E_{b \mathrm{~B}}\right) / k_{\mathrm{B}} T}+1\right]^{2}} d \varepsilon+ \\
& \left.+\frac{2 m_{\mathrm{p}}^{3 / 2}}{\sqrt{2} \pi^{2} \hbar^{3}} \frac{1}{\left(1+e^{\Delta^{*}(T) / k_{\mathrm{B}} T}\right)} \sqrt{\varepsilon_{\mathrm{F}}(T)}\right\} .
\end{aligned}
$$

In this case, the magnetic susceptibility is also defined as $\chi(T)=\chi_{\mathrm{t}}(T) M / \rho_{\mathrm{M}}$ for comparing with the experimental data [75]. It is weakly temperatureindependent (see Fig. 4), as observed experimentally in the optimally doped YBCO [75].

From the above considerations, it follows that the coexisting insulating and metallic phases are manifested in the temperature dependence of the magnetic susceptibility $\chi$ of underdoped to optimally doped cuprates.

\subsection{Temperature dependence \\ of the c-axis resistivity in high-T cuprates at different doping levels}

The analysis of the $c$-axis charge transport in high$T_{\mathrm{c}}$ cuprates at different doping levels may also provide the additional information about the insulating and metallic behaviors of these materials. The transport mechanisms in and out of plane are actually different. Here, we consider specifically the $3 \mathrm{D}$ polaron transport at the dissociation of interlayer large

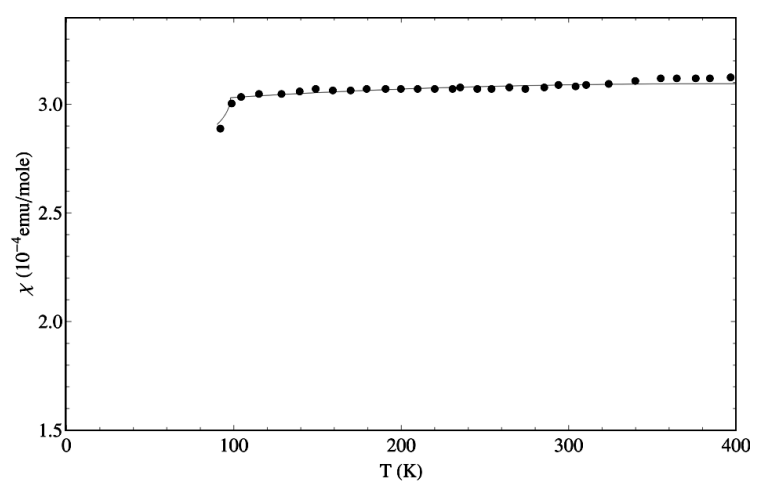

Fig. 4. Comparison of the theoretical results for the magnetic susceptibility of the high- $T_{\mathrm{c}}$ cuprate $\mathrm{YBa}_{2} \mathrm{Cu}_{3} \mathrm{O}_{6+x}$ (solid curve is obtained, by using the parameters $m_{\mathrm{p}}=2.1 m_{\mathrm{e}}$, $m_{\mathrm{I}}=4.1 m_{\mathrm{e}}, n_{\mathrm{D}}=0.05 \times 10^{19} \mathrm{~cm}^{-3}, \varepsilon_{\mathrm{F}}=0.18 \mathrm{eV}$, and $\varepsilon_{\mathrm{FI}}=0.148 \mathrm{eV}, \rho=5.9 \mathrm{~g} / \mathrm{cm}^{3}$, and $\left.E_{b \mathrm{~B}}=0.0001 \mathrm{eV}\right)$ with experimental data for $x=0.97(\bullet)$ [75]

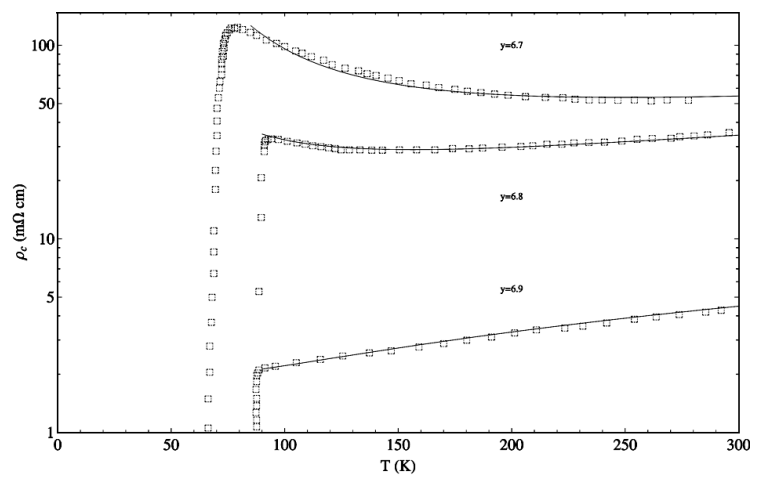

Fig. 5. The experimental $\rho_{\mathrm{c}}(T)$ data (dotted square) obtained for $\mathrm{YBa}_{2} \mathrm{Cu}_{3} \mathrm{O}_{y}$ at various doping levels [77] and the respective fits by Eq. (19) (solid lines). Fits to the experimental $\rho_{\mathrm{c}}(T)$ data for $\mathrm{YBa}_{2} \mathrm{Cu}_{3} \mathrm{O}_{y}$ with $y=6.7,6.8$, and 6.9 are performed, by using Eq. (19) and different fitting parameters $\left(\rho_{0}=0.05 \mathrm{~m} \Omega \mathrm{cm}, a_{h}=11.5 \times 10^{-8} \mathrm{~cm}, \omega_{0}=\right.$ $=5.8 \times 10^{13} \mathrm{~s}^{-1}, n_{i}=1.77 \times 10^{19} \mathrm{~cm}^{-3}, E_{b \mathrm{~B}}=0.0215 \mathrm{eV}$; $\rho_{0}=0.03 \mathrm{~m} \Omega \mathrm{cm}, a_{h}=11.5 \times 10^{-8} \mathrm{~cm}, \omega_{0}=6.0 \times 10^{13} \mathrm{~s}^{-1}$, $n_{i}=2.0 \times 10^{19} \mathrm{~cm}^{-3}, E_{b \mathrm{~B}}=0.0135 \mathrm{eV}$ and $\rho_{0}=0.02 \mathrm{~m} \Omega \mathrm{cm}$, $a_{h}=11.5 \times 10^{-8} \mathrm{~cm}, \omega_{0}=17.0 \times 10^{13} \mathrm{~s}^{-1}, n_{i}=7.35 \times$ $\times 10^{19} \mathrm{~cm}^{-3}$, and $\left.E_{b \mathrm{~B}}=0.005 \mathrm{eV}\right)$ for $y=6.7,6.8$, and 6.9 , respectively

bipolarons for studying the problem of insulator- tometal crossover in the bulk of cuprates. We assume that, in doped cuprates, the localized large bipolarons are formed in carrier-poor regions between the $\mathrm{CuO}_{2}$ layers, and the $c$-axis charge transport becomes possible at the thermal dissociation of these immobile bipolarons into separate polarons, which subsequently move, by hopping along the $c$-axis. According 
to Ref. [40], the $c$-axis resistivity of the cuprates above $T_{\mathrm{c}}$ can be determined as

$\rho_{\mathrm{c}}(T)=\rho_{0}+\frac{2 k_{\mathrm{B}} T}{n_{i} e^{2} a_{h}^{2} \omega_{0}} \exp \left[\frac{E_{b \mathrm{~B}}}{k_{\mathrm{B}} T}\right]$,

where $\rho_{0}$ is the residual resistivity, $a_{h}$ is the hopping distance, and $\omega_{0}$ is the out-of-plane optical phonon mode frequency.

Now, we will compare our predictions with the experimental $\rho_{\mathrm{c}}(T)$ data for $\mathrm{YBa}_{2} \mathrm{Cu}_{3} \mathrm{O}_{y}$ at various doping levels. In so doing, we apply the model based on the hopping conduction of polarons at the thermally activated dissociation of localized bipolarons residing between the $\mathrm{CuO}_{2}$ layers to the $c$-axis charge transport observed in $\mathrm{YBa}_{2} \mathrm{Cu}_{3} \mathrm{O}_{y}$. The comparison of our results with experimental $\rho_{\mathrm{c}}(T)$ data for various samples of $\mathrm{YBa}_{2} \mathrm{Cu}_{3} \mathrm{O}_{y}$ with different doping levels is shown in Fig. 5. One can see that the calculated results for $\rho_{\mathrm{c}}(T)$ agree very well with the experimental $\rho_{\mathrm{c}}(T)$ data down to $T_{\mathrm{c}}$. As can be seen by inspection of Fig. 5, the insulating behavior of $\rho_{\mathrm{c}}(T)$ in underdoped $\mathrm{YBa}_{2} \mathrm{Cu}_{3} \mathrm{O}_{y}$ is changed gradually to the metallic behavior with decreasing $E_{b \mathrm{~B}}$ in optimally doped regime, as shown in Fig. 5.

Our results show (see Fig. 5) that the binding energy of a bipolaron $E_{b \mathrm{~B}}$ decreases with increasing the concentration of polaronic carriers $n_{i}$ in carrier-poor regions between the $\mathrm{CuO}_{2}$ layers and becomes equal to zero at some critical value of $n_{i}$, at which the insulator-to-metal crossover would occur in YBCO. When $E_{b \mathrm{~B}}$ tends to zero, the insulating behavior of $\rho_{c}(T)$ changes to the metallic behavior in YBCO at $n_{i} \gtrsim 7.4 \times 10^{19} \mathrm{~cm}^{-3}$. With increasing the doping, large bipolarons begin to dissociate, and the insulator-to-metal transition occurs at high doping levels (i.e. at $y \gtrsim 6.9$ ), at which the binding energy $E_{b \mathrm{~B}}$ of such bipolarons becomes vanishingly small.

\section{Conclusions}

We have studied thoroughly the distinctive features of the metal-insulator transitions, multiscale phase separation, evolution of coexisting electronic phases (i.e., insulating and metallic/superconducting phases), and the possible manifestations of these interrelated phenomena in hole-doped cuprates. We have examined the effects of metal-insulator transitions and coexisting insulating, metallic, and superconducting phases on the normal and superconducting properties of the cuprates from the lightly to optimally doped regime, where the different types of localized and mobile hole carriers reside in hole-poor (insulating) and hole-rich (metallic) regions. We have argued that the charge carriers introduced into cuprates by hole doping are inhomogeneously distributed in various regions such as those in a deformable lattice and near the dopants (impurities) and other defects. The hole carriers interacting with the lattice vibrations become polaronic quasiparticles and segregate into insulating (carrierpoor) and metallic/superconducting (carrier-rich) regions as a result of their specific ordering.

We have shown that the metal-insulator transitions, multiscale phase separation into insulating, metallic, and superconducting stripes, and the evolution of the coexisting insulating and metallic/superconducting phases in hole-doped cuprates would occur in a wide doping interval from $x \simeq 0.02$ to $x=0.13$. Our results show that the narrow nanoscale metallic stripes in the lightly doped cuprates $(x \simeq$ $\simeq 0.02-0.05$ ) persist as vanishingly small islands between very large insulating regions, and then the coexistence of the competing macroscale insulating, metallic, and superconducting phases is expected in underdoped cuprates $(x \simeq 0.05-0.13)$. At $x>1 / 8$, the insulating stripes became narrower and persist as vanishingly small insulating islands, which finally disappear in the optimally doped cuprates. We have demonstrated that the metal-insulator transitions, multiscale phase separation, and coexisting insulating, metallic, and superconducting phases are manifested in the suppression of the critical superconducting transition temperature in underdoped high- $T_{\mathrm{c}}$ cuprates and in the unusual temperature-dependent behaviors of the magnetic susceptibility and the $c$-axis resistivity of lightly to optimally doped cuprates. We have determined the maximum values of the superconducting transition temperature $T_{\mathrm{c}}^{\max }$ in the optimally doped LSCO and YBCO. We have shown that the suppression of superconductivity in LSCO (at $x \lesssim$ $\lesssim 0.13$ ) and YBCO (at $x \lesssim 1 / 8$ ) is caused by an increase of the insulating volume fraction at the expense of the superconducting volume fraction, i.e., a decrease of the superconducting volume fraction is accompanied by the suppression of $T_{c}$. Further, we have found that $\chi(T)$ exhibits three distinctly different behaviors: (i) insulating behavior at $x \leq 0.05$, (ii) unusual metallic behavior in the underdoped regime $(x \approx 0.05-0.13)$, and (iii) usual metallic behavior 
in optimally doped regime. Similarly, the insulating behavior of $\rho_{\mathrm{c}}(T)$ in underdoped $\mathrm{YBa}_{2} \mathrm{Cu}_{3} \mathrm{O}_{y}$ is changed gradually to the metallic behavior in optimally doped $\mathrm{YBa}_{2} \mathrm{Cu}_{3} \mathrm{O}_{y}$ (see Fig. 5), when the binding energy $E_{b \mathrm{~B}}$ of large bipolarons residing between the $\mathrm{CuO}_{2}$ layers of the cuprates tends to zero at high doping levels. Most importantly, the distinctive features of the metal-insulator transitions, multiscale phase separation, evolution of the coexisting insulating and metallic/superconducting phases, and possible effects of these interrelated phenomena predicted by us are found experimentally in lightly to optimally doped cuprates.

We thank E.M. Ibragimova, P.J. Baimatov, Z.A. Narzikulov and E.X. Karimbaev for useful discussions. This work was supported by the Foundation of Uzbek Academy of Sciences, grant No. OT-Ф2-15.

1. M. Imada, A. Fujimori, Y. Tokura. Metal-insulator transitions. Rev. Mod. Phys. 70, 1039 (1998).

2. P.A. Lee, N. Nagaosa, X.-G. Wen. Doping a Mott insulator: Physics of high-temperature superconductivity. Rev. Mod. Phys. 78, 17 (2006).

3. S. Dzhumanov. Theory of Conventional and Unconventional Superconductivity in the High- $T_{c}$ Cuprates and Other Systems (Nova Science Publishers, 2013).

4. A.N. Lavrov, V.F. Gandmakher. Low-temperature resistivity of underdoped cuprates. Phys. Usp. 41, 223 (1998).

5. M.A. Kastner, R.J. Birgeneau, G. Shirane, Y. Endoh. Magnetic, transport, and optical properties of monolayer copper oxides. Rev. Mod. Phys. 70, 897 (1998).

6. T. Timusk, B. Statt. The pseudogap in high-temperature superconductors: an experimental survey. Rep. Prog. Phys. 62, 61 (1999).

7. S. Dzhumanov. The dependence of $T_{c}$ on carrier concentration in high- $T_{c}$ superconductors. Superlatt. Micros. 21, 363 (1997).

8. S. Dzhumanov, O.K. Ganiev, Sh.S. Djumanov. Pseudogap formation and unusual quasiparticle tunneling in cuprate superconductors: Polaronic and multiple-gap effects on the tunneling spectra. Phys. B 427, 22 (2013).

9. F. Walz. The Verwey transition - a topical review. J. Phys.: Condens. Matter 14, R285 (2002).

10. J. Bardeen, L.N. Cooper, J.R. Schrieffer. Theory of superconductivity. Phys. Rev. 108, 1175 (1957).

11. J. Zaanen. Self-organized one dimensionality. Science $\mathbf{2 8 6}$, 251 (1999).

12. C. Castellani, C. Di Casto, M. Grilli. Stripe formation: A quantum critical point for cuprate superconductors. J. Phys. Chem. Solids 59, 1694 (1998).

13. K.A. Müller, G.-M. Zhao, K. Conder, H. Keller. The ratio of small polarons to free carriers in $\mathrm{La}_{2-x} \mathrm{Sr}_{x} \mathrm{CuO}_{4}$ derived from susceptibility measurements. J. Phys.: Condens. Matter 10, L291 (1998).

ISSN 2071-0194. Ukr. J. Phys. 2019. Vol. 64, No. 4
14. N.L. Saini, A. Lanzara, A. Bianconi, D. Law, A. Menovsky, K.B. Garg, H. Oyanagi. Decrease of itinerant holes near the metal to insulator crossover in superconducting $\mathrm{La}_{1.85} \mathrm{Sr}_{0.15} \mathrm{CuO}_{4}$. J. Phys. Soc. Jpn. 67, 393 (1998).

15. S.A. Kivelson, I. Bindloos, E. Fradkin, V. Oganesyan, J. Tranquada, A. Kapitulnic, C. Howard. How to detect fluctuating stripes in the high-temperature superconductors. Rev. Mod. Phys. 75, 1201 (2003).

16. G. Campi, A. Bianconi, N. Poccia, G. Bianconi, L. Barba, G. Arrighetti, D. Innoceti, J. Karpinski, N.D. Zhigadlo, S.M. Kazakov, M. Burghammer, M.V. Zimmermann, M. Sprung, A. Ricci. Inhomogeneity of chargedensity-wave order and quenched disorder in a high- $T_{c}$ superconductor. Nature 525, 359 (2015).

17. A. Ino, C. Kim, M. Nakamura, T. Yoshida, T. Mizokawa, Z.-X. Shen, A. Fujimori, T. Kakeshita, H. Eisaki, S. Uchida. Electronic structure of $\mathrm{La}_{2-x} \mathrm{Sr}_{x} \mathrm{CuO}_{4}$ in the vicinity of the superconductor-insulator transition. Phys. Rev. B 62, 4137 (2000).

18. N.V. Anshukova, A.I. Golovashkin, L.I. Ivanova, A.P. Rusakov. The effect of superstructural ordering on the properties of high-temperature oxide superconductor systems. J. Exp. Theor. Phys 96, 1045 (2003).

19. S.I. Vedeneev. High-temperature superconductors in high and ultrahigh magnetic fields. Phys. Usp. 55, 625 (2012).

20. L. Forro. Out-of-plane resistivity of $\mathrm{Bi}_{2} \mathrm{Sr}_{2} \mathrm{CaCu}_{2} \mathrm{O}_{8+x}$ high temperature superconductor. Phys. Lett. A 179 (2), 140 (1993).

21. S. Ono, Yoichi Ando, T. Murayama, F.F. Balakirev, J.B. Betts, G.S. Boebinger. Metal-to-insulator crossover in the low-temperature normal state of $\mathrm{Bi}_{2} \mathrm{Sr}_{2-x} \mathrm{La}_{x} \mathrm{CuO}_{6+\delta}$. Phys. Rev. Lett. 85, 638 (2000).

22. T. Nakano, M. Oda, C. Manabe, N. Momono, Y. Miura, M. Ido. Magnetic properties and electronic conduction of superconducting $\mathrm{La}_{2-x} \mathrm{Sr}_{x} \mathrm{CuO}_{4}$. Phys. Rev. B 49, 16000 (1994).

23. S. Komiya, Y. Ando, X.F. Sun, A.N. Lavrov. c-axis transport and resistivity anisotropy of lightly to moderately doped $\mathrm{La}_{2-x} \mathrm{Sr}_{x} \mathrm{CuO}_{4}$ single crystals: Implications on the charge transport mechanism. Phys. Rev. B 65, 214535 (2002).

24. Sh. Sakita, F. Nakamura, T. Suzuki, T. Fujita. Structural transitions and localization in $\mathrm{La}_{2-x-y} \mathrm{Nd}_{y} \mathrm{Sr}_{x} \mathrm{CuO}_{4}$ with $p \sim 1 / 8$. J. Phys. Soc. Jpn. 68, 2755 (1999).

25. Y. Koike, M. Akoshima, M. Aoyama, K. Nishimaki, T. Kawamata, T. Adachi, T. Noji, M. Kato, I. Watanabe, S. Ohira, W. Higemoto, K. Nagamine, H. Kimura, K. Hirota, K. Yamada, Y. Endoh. Cu-site-substitution effects on the $1 / 8$ anomaly in the high- $T_{c}$ cuprates and on the anomaly at $x=0.21$ in $\mathrm{La}_{2-x} \mathrm{Sr}_{x} \mathrm{CuO}_{4}$. Phys. C 357-360, 82 (2001).

26. D. Pines. Spin fluctuations and $d_{x^{2}-y^{2}}$ pairing in the high temperature superconductors. Tr. J. Phys. 20, 535 (1996).

27. B.P. Stojkovic, D. Pines. Theory of the longitudinal and Hall conductivities of the cuprate superconductors. Phys. Rev. B 55, 8576 (1997).

28. A.A. Abrikosov. Resonant tunneling in high- $T_{c}$ superconductors. Phys. Usp. 41, 605 (1998). 
29. B. Sacépé, T. Dubouchet, C. Chapelier, M. Sanquer, M. Ovadia, D. Shahar, M. Feigel'man, L. Ioffe. Localization of preformed Cooper pairs in disordered superconductors. Nat. Phys. 7, 239 (2011).

30. B.K. Chakraverty, A. Avignon, D. Feinberg. Understanding high temperature superconducting oxides. J. LessCommon Metals 150, 11 (1989).

31. D. Emin, M.S. Hillery. Formation of a large singlet bipolaron: Application to high-temperature bipolaronic superconductivity. Phys. Rev. B 39, 6575 (1989).

32. J.T. Devrees, A.S. Alexandrov. Fröhlich polaron and bipolaron: Recent developments. Rep. Prog. Phys. 72, 066501 (2009).

33. S. Dzhumanov, P.J. Baimatov, A.A. Baratov, P.K. Khabibullaev. The continuum theory of delocalized and selftrapped polarons and bipolarons in solids. Phys. C 254, 311 (1995).

34. L.P. Gor'kov, A.V. Sokol. Phase stratification of an electron liquid in the new superconductors. JETP Lett. 46 (8), 420 (1987).

35. J. Zaanen, O. Gunnarsson. Charged magnetic domain lines and the magnetism of high- $T_{c}$ oxides. Phys. Rev. B 40, 7391 (1989).

36. V.J. Emery, S. Kivelson, O. Zachar. Spin-gap proximity effect mechanism of high-temperature superconductivity. Phys. Rev. B 56, 6120 (1997).

37. K.A. Müller. Recent experimental insights into HTSC materials. Phys. C 341, 11 (2000).

38. L.P. Gor'kov. Inherent inhomogeneity in two-component model for cuprates. J. Supercond. 14, 365 (2001).

39. V.V. Kabanov. Polarons: From single polaron to short scale phase separation. arXiv: cond-mat/0611174.

40. S. Dzhumanov, O.K. Ganiev, Sh.S. Djumanov. Normalstate conductivity of underdoped to overdoped cuprate superconductors: Pseudogap effects on the in-plane and $c$ axis charge transports. Phys. B 440, 17 (2014).

41. P.W. Anderson. Present status of the theory of the high- $T_{c}$ cuprates. Low Temp. Phys. 32, 282 (2006).

42. M. Eschring. The effect of collective spin-1 excitations on electronic spectra in high- $T_{c}$ superconductors. Adv. Phys. 55, 47 (2006).

43. C.M. Varma. High-temperature superconductivity: Mind the pseudogap. Nature 468, 184 (2010).

44. Yu. A. Nepomnyashchii, E.Ya. Pashitskii. Superfluid Bose liquid with intense bose pair condensate. JETP 98, 178 (1990).

45. V.L. Vinetskii, N.I. Kashirina, and E.A. Pashitskii. Bipolaron states in ion crystals and the problem of high temperature superconductivity. Ukr. J. Phys. 37, 76 (1992).

46. N.L. Kashirina, V.D. Lakhno, V.V. Sychyov. Correlation effects and Pekar bipolaron (arbitrary electron-phonon interaction). Phys. Stat. Sol. B 239, 174 (2003).

47. Ch.B. Lushchik, A.Ch. Lushchik. Decay of Electronic Excitations with Defect Formation in Solids (Nauka, 1989) (in Russian).
48. S. Dzhumanov, P.K. Khabibullaev. The coexistence of unstable, metastable, and separated Frenkel pair defects in solids. III. Theory of the non-impact mechanisms for defect formation in non-metals. Phys. Stat. Sol. B 152, 395 (1989).

49. S. Sugai. Local distortion specifying the superconductor phases observed by Raman scattering. Phys. C 185-189, 76 (1991).

50. F.M. Peeters, J.T. Devreese, G. Verbist. Possible (bi) polaron effects in the high- $t_{c}$ superconductors. Phys. Scrip. T 39, 66 (1991).

51. X.X. Bi, P.C. Eklund. Polaron contribution to the infrared optical response of $\mathrm{La}_{2-x} \mathrm{Sr}_{x} \mathrm{CuO}_{4+\delta}$ and $\mathrm{La}_{2-x} \mathrm{Sr}_{x} \mathrm{NiO}_{4+\delta}$. Phys. Rev. Lett. 70, 2625 (1993).

52. A. Ino, C. Kim, M. Nakamura, T. Yoshida, T. Mizokawa, A. Fujimori, Z-X Shen, T. Kakeshita, H. Eisaki, S. Uchida. Doping-dependent evolution of the electronic structure of $\mathrm{La}_{2-x} \mathrm{Sr}_{x} \mathrm{CuO}_{4}$ in the superconducting and metallic phases Phys. Rev. B 65, 094504 (2002).

53. D.N. Basov, T. Timusk. Electrodynamics of high- $T_{c}$ superconductors Rev. Mod. Phys. 77, 721 (2005).

54. S. Dzhumanov, P.J. Baimatov, O.K. Ganiev, Z.S. Khudayberdiev, B.V. Turimov. Possible mechanisms of carrier localization, metal-insulator transitions and stripe formation in inhomogeneous hole-doped cuprates. J. Phys. Chem. Solid. 73, 484 (2012).

55. M. Le Tacon, A. Bosak, S.M. Souliou, G. Dellea, T. Loew, R. Heid, K-P Bohnen, G. Ghiringhelli, M. Krisch, B. Keimer. Giant phonon anomalies and central peak due to charge density wave formation in $\mathrm{YBa}_{2} \mathrm{Cu}_{3} \mathrm{O}_{6.6}$ Nat. Phys. 10, 52 (2014)

56. E.M. Forgan, E. Blakburn, A.T. Holmes, A.K.R. Briffa, J. Chang, L. Bouchenoire, S.D. Brown, L. Ruixing, D. Bonn, W.N. Hardy, N.B. Christensen, M.V. Zimmermann, M. Hucker, S.M. Hayden. The microscopic structure of charge density waves in underdoped $\mathrm{YBa}_{2} \mathrm{Cu}_{3} \mathrm{O}_{6.54}$ revealed by X-ray diffraction. Nat. Commun. 6, 10064 (2015).

57. M. Miao, D. Ishikawa, R. Heid, M. LeTakon, G. Fabbris, D. Meyers. Incommensurate phonon anomaly and the nature of charge density waves in cuprates. Phys. Rev. X 8, 011008 (2018).

58. T. Kato, T. Noguchi, R. Saito, T. Machida, H. Sakata. Gap distribution in overdoped $\mathrm{La}_{2-x} \mathrm{Sr}_{x} \mathrm{CuO}_{4}$ observed by scanning tunneling spectroscopy. Phys. C 460-462, 880 (2007).

59. A.V. Puchkov, D.N. Basov, T. Timusk. The pseudogap state in high- $T_{c}$ superconductors: An infrared study. J. Phys: Condens. Matt. 8, 10049 (1996).

60. Yu.A. Izyumov, N.M. Plakida, Yu.N. Skryabin. Magnetism in high-temperature superconducting compounds. Usp. Fiz. Nauk. 159, 621 (1989).

61. J. Fink, N. Nucker, M. Alexander, H. Romberg, M. Knupeer, M. Merkel, P. Adelmann, R. Claessen, G. Mante, T. Buslaps, S. Harm, R. Manzke, M. Skibowski. High-

ISSN 2071-0194. Ukr. J. Phys. 2019. Vol. 64, No. 4 
energy spectroscopy studies of high- $T_{c}$ superconductors. Phys. C 185-189, 45 (1991).

62. S. Ono, Y. Ando, T. Murayama, F.F. Balakirev, J.B. Betts, G.S. Boebinger. Low-temperature normal state of $\mathrm{Bi}_{2} \mathrm{Sr}_{2-x} \mathrm{La}_{x} \mathrm{CuO}_{6+\delta}$ : Comparison with $\mathrm{La}_{2-x} \mathrm{Sr}_{x} \mathrm{CuO}_{4}$. Phys. C 357-360, 138 (2001).

63. A.L. Solovjov, H.-U. Habermeier, T. Haage. Fluctuation conductivity in $\mathrm{Y}-\mathrm{Ba}-\mathrm{Cu}-\mathrm{O}$ films with artificially produced defects . Fiz. Nizk. Temp. 28, 144 (2002) [Low Temp. Phys. 28, 99 (2002)].

64. A. Lanzara, P.V. Bogdanov, X.J. Zhou, S.A. Kellar, D.L. Feng, E.D. Lu, T. Yoshida, H. Eisaki, A. Fujimori, K. Kishio, J.-I. Shimoyama, T. Moda, S. Uchida, Z. Hussain, Z.-X. Shen. Evidence for ubiquitous strong electron-phonon coupling in high-temperature superconductors. Nature 412, 510 (2001).

65. P.W. Anderson. The Theory of Superconductivity in the High- $T_{\mathrm{c}}$ Cuprates (Princeton Univ. Press, 1997).

66. J.L. Tallon, J.W. Loram, J.R. Cooper, C. Panagopoulos, C. Bernhard. Superfluid density in cuprate high- $T_{c}$ superconductors: A new paradigm. Phys. Rev. B 68, 180501 (2003).

67. N. Ichikawa, S. Uchida, J.M. Tranquada, T. Niemöller, P.M. Gehring, S.-H. Lee, J.R. Schneider. Local magnetic order vs superconductivity in a layered cuprate. Phys. Rev. Lett. 85, 1738 (2000).

68. P.B. Allen, Z. Fisk, A. Migliori. Normal state transport and elastic properties of high- $T_{c}$ materials and related compounds. In: Physical Properties of High Temperature Superconductors I. Edited by D.M. Ginsberg (World Scientific, 1988), Chapter 5.

69. A.S. Alexandrov, V.V. Kabanov. Parameter-free expression for superconducting $T_{c}$ in cuprates. arXiv:condmat/9903071.

70. D.M. Eagles. Possible pairing without superconductivity at low carrier concentrations in bulk and thin-film superconducting semiconductors. Phys. Rev. 186, 456 (1969).

71. S. Dzhumanov, E.X. Karimboev, Sh.S. Djumanov. Underlying mechanisms of pseudogap phenomena and Boseliquid superconductivity in high- $T_{c}$ cuprates. Phys. Lett. A 380, 2173 (2016).

72. S. Dzhumanov, E.K. Karimboev. Competing pseudogap and impurity effects on the normal-state specific heat properties of cuprate superconductors. Phys. A 406, 176 (2014).

73. M. Houssa, M. Ausloos. Thermal conductivity of high- $T_{c}$ superconductors: effect of Van Hove singularities. Phys. $C$ 265, 258 (1996).

74. L. Ping. Possible origin of the broad peak around $450 \mathrm{~cm}^{-1}$ of the c-axis optical conductivity of the underdoped $\mathrm{YBa}_{2} \mathrm{Cu}_{3} \mathrm{O}_{6+x}$ in the superconducting state. Phys. Rev. $B$ 65, 214511 (2002).
75. J.W. Loram, K.A. Mirza, J.R. Cooper. Properties of the superconducting condensate and the normal state pseudogap in high $T_{c}$ cuprates derived from the electronic specific heat. IRC Res. Rev. 3, 77 (1998).

76. M. Roulin, B. Revaz, A. Junod, A. Erb, E. Walker. High resolution specific heat experiments on the vortex melting line in $\mathrm{MBa}_{2} \mathrm{Cu}_{3} \mathrm{O} x(\mathrm{M}=\mathrm{Y}$, Dy and $\mathrm{Eu})$ crystals: Observation of first- and second-order transitions up to $16 \mathrm{~T}$. In: Physics and Materials Science of Vortex States, Flux Pinning and Dynamics. Edited by R. Kossowsky, S. Bose, Z. Durusoy, V. Pan, (Springer, 1999), p. 489.

77. S. Tajima, J. Schützmann, S. Miyamoto et al. Optical study of c-axis charge dynamics in $\mathrm{YBa}_{2} \mathrm{Cu}_{3} \mathrm{O}_{y}$ :nn Carrier self-confinement in the normal and the superconducting states. Phys. Rev. B 55, 6051 (1997).

Received 12.02.19

С. Джуманов, I. Хідіров,

У.Т. Курбанов, З.С. Худайбердієв, Дж.Ш. Рашідов

ОСОБЛИВОСТІ ПЕРЕХОДІВ

МЕТАЛ-ІЗОЛЯТОР, БАГАТОШАРОВЕ

ФАЗОВЕ РОЗДІЛЕННЯ ТА ПОВ'ЯЗАНІ ЕФЕКТИ

В КУПРАТАХ, ЛЕГОВАНИХ ДІРКАМИ

$\mathrm{P}$ е з ю м е

Вивчаються особливості переходів метал-ізолятор, багатошарове фазове розділення та еволюція співіснуючих ізоляційних та металевих/надпровідних фаз у купратах, легованих дірками. Ми показуємо, як ці взаємопов'язані явища і пов'язані з ними ефекти проявляються в широкому діапазоні від низького до оптимального легування цих систем, де локалізовані і рухомі носіі дірок знаходяться в бідних (ізолюючих) і багатих на дірки (металеві або надпровідні) областях. Ми стверджуємо, що невеликі ділянки багаті на дірки (тобто вузькі нанорозмірні металеві острови або смуги) можуть зберігатися в ізолюючій фазі слабо легованих купратів, тоді як конкуруючі ізоляційні, металеві та надпровідні фази будуть співіснувати в слабо легованих купратах. Коли рівень легування збільшується далі, ділянки бідні на дірки (або ізолюючі зони) поступово звужуються від макро- до нанорозмірних ізолюючих смуг і зникають в оптимально легованих купратах. Наочно продемонстровано, що переходи метал-ізолятор і співіснуючі ізоляційні та металеві/надпровідні фази проявляються в пригніченні надпровідності в слабо легованих купратах та в різній температурно-залежній поведінці магнітної сприйнятливості і опору в напрямку с-осі для купратів в інтервалі від низького до оптимального рівня легування. 